\title{
Somos inevitablemente morales
}

- Adela Cortina

Para empezar la tarea de averiguar qué es ser una persona moralmente educada, resulta de gran utilidad recurrir a una tradición española que, iniciada por Xavier Zubiri y proseguida por José Luis Aranguren, continúa hoy su andadura en trabajos como los de los miembros del Seminario Xavier Zubiri, y muy especialmente en los de su director, Diego Gracia. Entiende la mencionada tradición que, para hablar de ética, es preciso acudir en principio a la antropología biológica con objeto de descubrir cuáles sean las raíces antropológicas de la moralidad, porque es imposible dar razón del fenómeno moral sin preguntarse por el modo de estar del ser humano en el mundo. A esta tarea dedicó Xavier Zubiri algunos de sus cursos orales' y Aranguren tuvo buen cuidado de aprovechar tales enseñanzas en su Ética ${ }^{2}$. Por su parte, Diego Gracia recoge y profundiza la "fundamentación" zubiriana de lo moral en lugares centrales de sus Fundamentos de Bioética", y otros autores hispanohablantes e "hispanoescribientes" le han dedicado sustanciosos comentarios ${ }^{4}$. A todos estos trabajos vamos a remitirnos con el objeto de dilucidar si nuestra misma estructura biológica exige alguna línea de educación moral, línea cuyos trazos iremos dibujando a lo largo del capítulo.

Atendiendo a esta tradición, podemos decir que todo ser humano se ve obligado a conducirse moralmente, porque está dotado de una "estructura moral" o, por decirlo con Diego Gracia, de una "protomoral", que tiene que distinguirse de la "moral como contenido". Precisamente porque todo ser humano posee esta estructura, podemos decir que somos constitutivamente morales: podemos comportarnos de forma moralmente correcta en relación con determinados contenidos morales, o bien de forma inmoral con respecto a ellos, pero, estructuralmente hablando, no 
existe ninguna persona que se encuentre situada "más allá del bien y del mal”. ¿En qué consiste esa estructura moral?

\section{La estructura moral del ser humano. Necesitamos sobrevivir}

En principio, recuerda Zubiri que cualquier organismo se ve enfrentado al reto de ser viable en relación con su medio y para ello se ve obligado a responder a las provocaciones que recibe de él ajustándose para no perecer. La estructura básica de la relación entre cualquier organismo y su medio es entonces "suscitación-afección-respuesta", y es la que le permite adaptarse para sobrevivir. Sin embargo, esta estructura se modula de forma bien diferente en el animal y en el ser humano. En el animal, la suscilación procede de un estímulo que provoca cn él una respuesta perfectamente ajustada al medio, gracias a su dotación biológica. A este ajustamiento se denomina "justeza" y se produce de forma automática. En el ser humano, sin embargo, en virtud de su hiperformalización, la respuesta no se produce de forma automática, y en esta no determinación de la respuesta se produce el primer momento básico de la libertad. Y no sólo porque la respuesta no viene ya biológicamente dada, sino también porque, precisamente por esta razón, se ve obligado a justificarla.

\section{El momento básico de la libertad}

El ser humano responde a la suscitación que le viene del medio a través de un proceso en el que podríamos distinguir tres pasos: (1) En principio, se hace cargo, a través de su inteligencia, de que los estímulos son reales, es decir, que proceden de una realidad estimulante por la que se siente afectado. El ser humano no está afectado, por tanto, por el "medio", sino por la realidad, lo cual supone un compromiso originario con ella que tendrá, como veremos, sus implicaciones morales. (2) La respuesta no le viene dada de forma automática, sino que, a la hora de responder, crea él mismo un conjunto de posibilidades, entre las que ha de elegir la que quiere realizar. Si bien tales posibilidades enraízan en la realidad, ellas mismas son irreales y es la persona quien tiene que elegir cuál quiere realizar. De ahí que los representantes de la tradición que estamos comentando convengan en afirmar que ya en ese nivel biológico básico se produce el primer momento de libertad: no estamos determinados por el estímulo real, sino que nos vemos forzados a elegir. No somos libres para dejar de ser libres.(3) Para elegir una posibilidad, el ser humano ha de renunciar a las demás y por eso su elección ha de ser justificada; es decir, ha de hacer su ajustamiento a la realidad, porque no le viene dado naturalmente, justificándose. Lo que en el animal era justeza automática, en el ser humano es justificación activa, y esta necesidad de justificarse le hace necesariamente moral. El "contenido" desde el cual una persona justificará sus elecciones no importa ahora, porque sin duda variará; lo que importa es recordar que se siente afectada por la realidad y para sobrevivir ha de 
responder a ella, eligiendo entre posibilidades y justificando su elección. ¿Qué se sigue de todo ello para la educación moral?

\section{Consecuencias para la educación moral}

En principio, si cualquier persona capta las cosas como "realidades" y su modo de estar en el mundo es el de estar en la realidad, necesitamos hacer pie en ella para construir cuantas posibilidades seamos capaces de idear; posibilidades entre las cuales tenemos que elegir la que consideremos más adecuada. Este sencillo reconocimiento comportará un buen número de implicaciones para quien desee ser una persona moralmente bien educada, aunque para extraerlas emplearemos el término "realidad" en un sentido un tanto heterodoxo en lo que a la tradición zubiriana se refiere. La primera de esas implicaciones consiste en reconocer que una persona inteligente trata de conocer la realidad.

\subsection{Conocer la realidad}

Si de la realidad tenemos que partir inevitablemente, parece aconsejable tratar de conocerla lo mejor posible, porque otra cosa es, no sólo suicida, sino también "homicida". Es suicida, ya que quien construye castillos en el aire, desconociendo el terreno que pisa, no tarda en caer en algún hoyo. La imaginación pueril, como es sabido, es la que se pierde en ensoñaciones sin punto de apoyo en la realidad, y después sucede que el niño, a fuerza de imaginar sin fundamento, se creyó Tarzán y se rompió el fémur pensando que saltaba de liana en liana. La imaginación creadora, por el contrario, la imaginación adulta, es la que se nutre de la realidad y trata de ampliarla proyectando desde ella. Por otra parte, desconocer la realidad y construirse la vida de espaldas a ella es también "homicida": el que vive siempre en las nubes es un peligro privado y público, bien porque propone proyectos ilusorios, que acaban en la frustración de quienes se alistaron confiados, bien porque se ignora si está causando daño o bien. Su ignorancia resulta, en cualquier caso, peligrosa. Por eso conviene experimentar la realidad cotidiana, informarse, recurrir a las aportaciones de distintos saberes, y echar mano de la experiencia ajena a través de la literatura, el cine, las artes plásticas y unos medios de comunicación "bien administrados". Una mente abierta a los problemas y a las propuestas de solución ya existentes es esencial para una persona moralmente educada.

\subsection{Tomarla en serio}

Ahora bien, precisamente quien se afane por conocer la realidad al máximo caerá en la cuenta de que con ella no se pueden hacer mangas y capirotes como a veces cree un "idealismo" mal entendido. Y digo "un idealismo malentendido", porque el término "idealismo" tiene diversas acepciones, algunas de las cuales son imprescindibles para culminar con éxito el proceso de humanización 
de la humanidad, micntras que otras resultan totalmente contraindicadas para llevar a cabo ese proceso. Sería idealismo positivo considerar que la historia humana sc construyc también con ideas ideales, y que es puro conformismo, Jcjación de humanidad, resignarse a pensar que no hay más cera que la que ardc, alerrarse con uñas y dientes a la vulgaridad y la ramplonería, tratando de ilusos a cuantos intentan abrir nuevos horizontes. Es también positivo el idealismo filosófico de cortc kantiano, según el cual, cuando hacemos ciencia, somos nosotros los que construimos modelos y plantamos preguntas que después formulamos a la expcriencia. Una teoría científica no es nunca un simple reflejo de la realidad, sino que cuenta, como ingrediente, con una buena dosis de imaginación y razón creadoras. Sin embargo, cuando extremamos estas posiciones y llegamos a afirmar que somos nosotros quienes construimos el mundo a nuestro sabor, desde nuestras ideas, sin necesidad siquiera de hacer pie en la realidad, hemos convertido el idealismo sano en un idealismo patológico. Y como sucede que todo conocimiento viene movido por un interés, como ya recordaron Scheller, Apcl o Habcrmas`, las más de las veces este idealismo enfermo está manipulado desde intcrescs individuales o grupales: interesa creer que somos nosotros quienes construimos la realidad porque así podemos manipularla a nuestro gusto. Éste es el caso ejemplar de la célebre "razón instrumental" moderna, que se viene empeñando desde hace siglos en tratar como objetos de usar y tirar a la naturaleza y a cada uno de los seres humanos ${ }^{6}$. Así hemos llegado en nuestro momento, sin ir más lejos, a una manipulación total del medio ambiente, gracias a la cual puede hablarse, sin temor a errar, de desastre ecológico. La desertización del planeta Tierra es ya un hecho, y al menos cincuenta millones de "ecorrefugiados" están abandonando sus países de origen, totalmente expoliados por intereses económicos, y buscan donde vivir. Es difícil calcular el número de muertcs que se producen por el camino. Es difícil saber qué ocurrirá cuando lleguen a la puerta de los "países ricos". Y no es mucho más sencillo predecir qué sucederá con los que logren entrar en ellos: marginación, busca de permiso de trabajo, Jcsconfianza y recelos por parte de los ciudadanos de toda la vida. Por el contrario, lo que sí es evidente es que la realidad empieza a "vengarse" con el agotamiento de las materias primas, la desertización y la progresiva destrucción de la ccosfera, porque la realidad, al cabo, termina "vengándose" de alguna manera. Termina presentando repleto su libro de reclamaciones a una humanidad que ha pcrdido el norte. Frente a esta idea errada de que podemos tomar la realidad a beneficio de inventario, puede extraerse una segunda consecuencia del reísmo zubiriano: no podemos organizar nuestra vida de espaldas a la realidad, sino que hemos de tomarla en serio.

\subsection{La imaginación al poder}

Pero tomarla en serio significa, entre otras cosas, saber que puede ser ampliada de una forma prodigiosa, siempre que no dejemos de hacer pie en ella y 
mientras agudicemos al máximo la capacidad creadora para imaginar posibilidades sin cuento. Como antes hemos comentado, es un hábil recurso de los conformistas, de los "realistas" de pacotilla frenar cualquier propuesta innovadora alegando que no hay más cera que la que arde, que no hay que estirar el brazo más que la manga, y otros refranes disuasorios. Sin embargo, lo humano, lo realmente humano, consiste en la capacidad de crear más cera y alargar las mangas, para conseguir la luz que deseamos y tener el brazo a cubierto. Las más de las veces, las depresiones, los malos humores, vienen motivados por el agobio que produce no vislumbrar salidas para nuestros problemas. Por eso, la piedra filosofal para salir del mal paso, para convertir en serenidad la amargura, consiste en idear alternativas viables. Una cosa es soñar utopías cuyo fracaso conduce a la frustración de los ideales por los que nacieron, otra bien distinta, ampliar el ámbito de la realidad posible, para encontrar siempre, ante cualquier problema, una salida. Los enigmas excitan la imaginación y la razón creadoras; las aporías, los callejones sin salida, bloquean las capacidades humanas y acaban matando el impulso vital.

\section{Ser realistas}

Actuar en el sentido que venimos comentando es, en definitiva, ser realista de un modo plenamente humano: ser realista en el sentido en que lo entendió un zubiriano como Ignacio Ellacuría, cuyo planteamiento moral y ético tuvo un agudo sentido de la realidad'. Por "ser realista" suele entenderse en principio ser pragmático, ser egoísta, no comprometer demasiado para no tener sinsabores. Pero esto no es realismo, sino cinismo. También puede entenderse por "realismo" atenerse a la realidad tal como es, a los hechos tal como son. Pero sucede que "lo que es, no es todo", sino que puede ser muchísimo más, dependiendo de nuestra "ambición". Por eso consideramos aquí auténtico realismo el que, al formular las grandes preguntas éticas, trata de ampliar lo real desde lo que ya es. Y hoy en día - fuerza es reconocerlo- no es realista preguntarse "¿qué hacer?" sólo desde el Primer o el Segundo Mundo, prescindiendo del Tercero, porque no existe ya ningún mundo que sea independiente de los restantes. Como muestra la globalización de los problemas económicos o ecológicos, la interdependencia cntre todos los lugares de la Tierra es un hecho, y para ser realista, para actuar con sentido de la realidad es preciso preguntarse qué hacer desde el contexto de la humanidad en su conjuntox. Por eso, los planteamientos éticos que parten de la psicología de cada individuo y tratan de llegar a partir de ella a la conveniencia para cada uno de aceptar determinadas propuestas morales carecen de realismo, porque no existen individuos abstractos, independientes de los pueblos, las culturas y las épocas. Cada uno de nosotros es hijo de su tiempo y de su lugar, scncillamentc porque devenimos personas a través de un proceso de socialización, en el que recibimos el legado de tradiciones de nuestra sociedad concreta. Por lo tanto, el punto de partida de la moralidad no es nunca un individuo cualquiera, sino este individuo en este grupo social, en esta época y en este 
lugar. Recordar que la pregunta por el hacer humano siempre se formula desde una situación fue una de las claves de la ética realista de la liberación de Ignacio Ellacuría.

\subsection{Un imperativo ético}

Una simple mirada a la globalidad — recuerda Ellacuría - nos muestra que existen "pueblos enteros crucificados", al menos dos tercios de la humanidad, lo cual significa que partimos ya de una situación de "deshumanidad". Un proyecto ético no puede eludir este punto de partida, sino intentar que el proceso evolutivo de hominización, por el que fue surgiendo paulatinamente el ser humano, se prolongue en un proceso de humanización. Para lograr llevar a cabo este proceso de humanización, quien quiera ser libre ha de asumir un "imperativo ético" que se articula en tres momentos: hacerse cargo de la realidad, cargar con ella y encargarse de ella para que sea como debe ser. "Hágase usted cargo" —decimos cuando pretendemos que alguien comprenda bien una situación antes de decidir, no sea cosa que tome una decisión de la que después pueda arrepentirse. "Cargue usted con las consecuencias", decimos cuando queremos mostrar a alguien que es a él a quien van a pedirse responsabilidades de lo que sucede, porque es quien toma la decisión, por mucho que quiera escabullir el bulto. "En definitiva es usted el encargado", decimos cuando el responsable se esfuerza por pasar la pelota al superior, al inferior o al sistema en su conjunto. Asumir estas tres obligaciones con la realidad social en la que ya estamos implantados es lo contrario de encogerse de hombros alegando que, a fin de cuentas, "no soy guardián de mi hermano". Pero en este caso no sería Yahvé quien pediría responsabilidades, sino la realidad de un ingente número de seres humanos despojados de sus derechos, ante el que es preciso haber perdido mucha humanidad para no sentirse conmovido, es preciso haber perdido mucha inteligencia para no comprender que las cosas deben ser de otro modo y que son también responsabilidad nuesIra. Curiosamente, Occidente se vanagloria de tener por justas las leyes que enunciamos poniéndonos en el lugar de cualquier otro. "Póngase usted en mi lugar", decimos al administrativo que nos pide lo imposible. "Póngase usted en mi lugar", repetimos en situaciones de desesperación. Ponerse en el lugar del dominicano agredido o del turco humillado es un buen comienzo para juzgar después si el racismo es humanamente aceptable.

\subsection{Ser responsables}

Desde esta perspectiva se abre paso lo que llamaríamos una "moral de la responsabilidad", entendida ahora no tanto en sentido weberiano como contraposición a la moral de la convicción", sino como contrapartida de la "moral de la irresponsabilidad". Porque el que intenta eludir a la realidad y no responder de ella, como si no presentara sus exigencias ni tuviera relación con él, practica una moral de la irresponsabilidad, que a la larga termina pagándose. Y digo el impersonal "se" 
con plena conciencia, porque, lamentablemente, no siempre es el irresponsable quien paga las malas consecuencias, sino otros más débiles que él. No es extraño, en este orden de cosas, que Ignacio Ellacuría hiciera suya esa moral de la responsabilidad de que vengo hablando: dejarse afectar por la realidad, hacerse cargo de ella y responder de ella, hasta el punto de introducir en el Plan de Estudios de la Universidad Centroamericana "José Simeón Cañas" una asignatura llamada "Realidad Nacional". Ni resu.lta extraño tampoco que el filósofo Hans Jonas propusiera hace bien poco sustituir el "Principio Esperanza", del marxista utópico Ernst Bloch, por un "Principio Responsabilidad", con respecto a la naturaleza".

\subsection{Ser inteligentes}

Si, como hemos dicho, vamos a vernos obligados constantemente a elegir entre posibilidades para apropiarnos unas, renunciando a otras, $y$ a tener que justificar nuestras elecciones, más nos vale intentar ir aclarándonos a nosotros mismos desde dónde hacerlo para lograr buenas elecciones. Sin duda, nuestra razón tiene una función calculadora, pero nos conviene que esos cálculos sean prudentes". Es decir, nos conviene tener criterios fundados desde los que tomar las decisiones, no sea que elijamos las opciones menos acertadas. En este sentido, la historia de la ética ha sido en buena medida la historia de la sabiduría moral, el intento de pertrechar a las personas de los criterios necesarios para hacer buenos juicios y, consiguientemente, buenas elecciones. Ejercitar la capacidad de juzgar para tomar decisiones prudentes es, pues, otro de los rasgos imprescindibles en la educación moral. ¿Desde dónde pueden hacerse las elecciones? La respuesta de Zubiri ante esta cuestión es básicamente la siguiente: en principio, cada hombre está dotado de unas tendencias inconclusas que lo llevan a preferir unas posibilidades, a considerarlas deseables, y son esas tendencias las que justifican sus preferencias $y$, por tanto, sus elecciones. Tales tendencias proceden fundamentalmente de la constitución temperamental de cada persona, que le vicne dada por nacimiento; de los ideales de hombre y los códigos morales vigentes en la sociedad de su época, y del nivel de desarrollo moral alcanzado tanto por la persona como por la sociedad en que vive. Es decir, ante todo, de lactores temperamentales y sociales, que son sin duda variables. Todos estos rasgos pertenecen todavía a una protomoral, pero de ellos podemos extraer también consecuencias para la educación moral, porque una persona moralmente bien educada tiene que ser consciente de la variabilidad de los contenidos morales y superar, ya desde esta perspectiva, cualquier tipo de dogmatismo.

\section{El dogmatismo es irracional}

La convicción de que en el terreno moral existen unos contenidos incontrovertibles, válidos para todo tiempo, y que sobre ellos no se puede discutir siquiera, es desafortunada. Y no sólo porque tiene malas consecuencias, dado que cl dogmático se convierte frecuentemente en fanático. Como decía Ortega, "la 
definición de la verdad por la utilidad es la definición de la mentira" y, por tanto, no se trata de descartar el dogmatismo sólo por sus consecuencias funestas, sino sobre'todo porque se cierra a la discusión. Cualquier persona con un mínimo de sentido común reconoce, por el contrario, que los contenidos morales han ido cambiando históricamente y que tampoco ahora mismo los diversos grupos humanos tienen por morales los mismos contenidos. Por eso es importante estar abierto a discutir las propias posiciones; lo cual no significa que de la discusión vaya a seguirse cambiar de contenidos, sino, en muchas ocasiones, conlirmar aquellos que se tenían por morales. Por eso, lo que resulta más desafortunado en el caso del dogmático es su negativa a entrar en un diálogo. La disposición a dialogar es, por el contrario, la que nos permite superar el dogmatismo, pero también el particularismo y el relativismo, igualmente insostenibles.

\section{El relativismo es inhumano}

De la diversidad de contenidos morales en el tiempo y en el espacio no cabe extraer como consecuencia la bondad del particularismo y del relativismo. La ley del péndulo es una ley que funciona históricamente, pero las más de las veces resulta inadecuada. Consistiría el particularismo en creer que entre las distintas formas de vida de los diferentes pueblos no existe ninguna sintonía, de suerte que "diversidad de contenidos morales" significaría "separación tajante" entre unas formas de vida y otras, e imposibilidad de hallar algunos elementos comunes a todas ellas. A un particularismo semejante podría acabar conduciéndonos un comunitarismo que, llevado por su entusiasmo por la comunidad, tuviera por normas de justicia únicamente las vigentes en ella ${ }^{12}$. Si el particularismo se toma en serio, entonces resulta imposible establecer un diálogo sobre cuestiones morales entre diferentes culturas, porque entre dos interlocutores que no tienen nada en común, no puede haber un diálogo. Y, sin embargo, vemos cómo uno de los rasgos peculiares de nuestro tiempo es el diálogo intercultural, que está resultando bien fructífero, por cierto. Por su parte, el relativismo no pone en duda quc existan valores "valiosos", normas válidas o ideales atractivos, sólo que declara que la calificación moral de una acción como buena o mala depende de cada cultura o de cada grupo. En el ámbito moral — según el relativista - no hay nada universal, sino que el reconocimiento de una acción como buena o mala, o de un valor como valioso o anodino, es relativo a cada cultura concreta y a cada grupo determinado. La verdad es que el relativismo es humanamente insostenible, como sc ccha de ver en nuestras sociedades que sólo de palabra son relativistas. Abochornados por nucstro inveterado etnocentrismo, nos parece a los europeos de mala educación, autoritario y dogmático, decir que no todas las opiniones son igualmentc respetables y alirmamos que tan racional es cualquier cultura como la nuestra, $\mathrm{e}$ incluso mucho más. Pero, cn realidad, si recordamos el caso de Salman Rushdie, nos parece irracional que una cultura condene a muerta a un ciudadano por escribir un libro, por muy blasferno que sea; nos indigna el trato dado a las mujeres en 
esa misma cultura; desaprobamos el sistema de castas de la India, e incluso afirmamos que los fundamentalistas del fis "todavía" andan por la Edad Media y "aún"no han llegado a la Modernidad, como si todos tuvieran que seguir nuestro mismo proceso. Y es que a la hora de la verdad, el relativismo no se lo cree nadie. Quien tiene por imacional quitar la vida, dañar física y moralmente, privar de libertades o no aportar los mínimos materiales y culturales para que las personas desarrollemos una vida digna, no lo cree sólo para su sociedad, sino para cualquiera. Y, lo diga o no, considera que una cultura indiferente al respeto a esos mínimos está ella misma bajo mínimos de racionalidad o, lo que es idéntico, de moralidad. Podemos decir, por tanto, que entre la Scila del dogmatismo y la Caribdis del relativismo, entre el rechazo del diálogo por innecesario (dogmatismo) y el rechazo por imposible (relativismo), se sitúa el justo medio, el quicio de una buena educación.

\section{Notas}

1. Zubiri, X. Sobre el hombre, Alianza, Madrid, 1986, sobre todo los capítulos I y vil.

2. López Aranguren, J.L. Ética, parte I, capítulo VII.

3. Gracia, D. Fundamentos de Bioética, Eudema, Madrid, 1988, pp. 366 y siguientes.

4. Pintor Ramos, A. Verdad y sentido, Universidad Pontificia de Salamanca, 1993. Conill, J., La ética de Zubiri, en El Ciervo, números 507-509 (1993), pp. 10 y 11 . Por mi parte, modestamente me he permitido ocuparme de esta ética en Ética sin moral, Tecnos, Madrid, 1990, pp. 55 y ss.

5. Scheller, M., Sociología del saber, Siglo XX, Buenos Aires, 1973, pp. 7681. Apel, K.-O., Ciencia y técnica como ideología, Tecnos, Madrid, 1984; Conocimiento e interés, Taurus, Madrid, 1984; Cortina, A., Ética y utopía: La Escuela de Frankfurt, pp. 115-119.

6. Como decía M. Horkheimer, criticando que la razón instrumental se haya convertido para Occidente en "la" razón: "Hoy se considera que la tarea, e incluso la verdadera esencia de la razón, consiste en hallar medios para lograr los objetivos propuestos en cada caso. Los objetivos que, una v4ez alcanzados, no se convierten ellos mismos en medios, son considerados como supersticiones". Ver Crítica de la razón instrumetnal, Sur, Buenos Aires, 1969. p. 7.

7. Ellacuría, I., Ética fundamental, curso inédito, San Salvador, 1978. La función liberadora de la filosofía en Escritos políticos, I, San Salvador, 1991.

8. Tortosa, J. Ma, Sociología del sistema mundial, Tecnos, Madrid, 1992.

9. Weber, M., Política como vocación, en El político y el científico, pp. 81-179.

10. Jonas, H. El principio de responsabilidad. Ensayo de una ética para la civilización tecnológica, Círculo de Lectores, Barcelona, 1994.

11. Rescher, N. La racionalidad, Tecnos, Madrid, 1994.

12. MacIntyre, A. Is Patriotism a Virtue?, The Lindley Lectures, University of Kansas, 1984. 